\title{
Mitigation of Rain Impact on Microwave Backhaul Networks
}

\author{
Forough Yaghoubi ${ }^{\dagger}, \mathrm{Jiaji}_{\mathrm{C}} \mathrm{Chen}^{\dagger}$, Ahmad Rostami $^{\ddagger}$, and Lena Wosinska ${ }^{\dagger}$ \\ ${ }^{\dagger}$ Optical Networks Laboratory, Communication Systems, KTH Royal Institute of Technology, 164 40, Stockholm, Sweden \\ ${ }^{\ddagger}$ Ericsson Research, 16483 Stockholm, Sweden \\ Emails: forough@kth.se, jiajiac@kth.se,wosinska@kth.se, and ahmad.rostami@ericsson.com
}

\begin{abstract}
Microwave backhaul networks are a cost-efficient option to support increasing capacity demands of mobile networks. However, inherent vulnerability of wireless backhauling to random fluctuations of the wireless channel complicates the design of reliable backhaul links. Long-lasting channel fluctuations such as rain fading may bring significant network performance degradation, and therefore, need to be carefully treated. This paper proposes a novel rain detection algorithm utilizing both temporal and spatial correlation of link status, aiming at efficiently distinguishing between long-term and shortterm channel fading. With this distinction, a central controller decides whether network-wide strategies, such as rerouting, are required to mitigate the effects of rain. The accuracy of the proposed detection method is evaluated by measuring false alarm and misdetection probabilities. Numerical results show high rain detection accuracy of the proposed algorithm. Consequently, the impact of imperfect rain detection on the network throughput performance and on the overhead imposed to the central controller becomes negligible.
\end{abstract}

Index Terms-Rain detection, microwave backhauling, SDN, millimeter wave communications, $5 \mathrm{G}$.

\section{INTRODUCTION}

The ever-growing traffic demand in mobile networks creates the need for improvement of the area spectral efficiency using ultra dense base station deployments. This densification challenges the reliability, scalability, and cost efficiency of the existing cable-based backhaul solutions. There is a growing consensus in both academia and industry that wireless technology will play an important role in backhauling of next generation radio access networks, referred to as 5G [1]. Wireless backhauling, however, entails vulnerability to fluctuations of the wireless channel attenuation, especially at submillimeter and millimeter waves, where the current wireless backhaul links operate [2]. Link adaptation strategies such as Adaptive Modulation and Coding (AMC) have already been applied in the commercial systems to compensate for the stochastic behavior of the channel attenuation [3]. It adjusts the transmission rate at the physical layer to maintain a certain quality of service level, which is usually defined in terms of Bit-Error-Rate (BER) [3]. The main events that may trigger the link adaption include rain, fog, snow, multipath fading, path obstruction, and interference. These events can affect the network for either short or long period, which requires different actions to efficiently mitigate their negative effects. Short events such as multipath fading need fast response, and therefore, are better to be handled locally on the link level, while long-lasting events, such as rain, path obstruction and interference, can stay for a relatively long period (e.g., half an hour and even more), where link-level adaptations are not sufficient. It is because the reduced link capacity caused by the long-lasting events may dramatically impact several important network performance metrics, e.g., resulting in low throughput and long delay. Hence, these events should be handled at the network layer (e.g., by rerouting) to avoid significant network performance degradation. To obtain an efficient network-layer adaptation, the first step is to accurately identify the types of events, namely short- or long-lasting change.

Most of the existing backhaul networks have a star or ring topology [4], while (partial) mesh topology is becoming more attractive, as it offers higher network capacity and higher survivability, as well as better load balancing [5]. Mesh topology enables rerouting and flow control at the network level, which requires a global view of the network. Collecting such global information, however, may be overwhelming since it requires a periodical collection of all channel information from each node/link [5], making it applicable only for the longlasting changes in the channel gains. Furthermore, Central Controller (CC) is needed to decide, based on the global information, whether or not to perform rerouting. Software Defined Networking (SDN), as an indisputable solution of 5G control plane, makes it possible to have an efficient $\mathrm{CC}$ [6].

There is a growing interest in using microwave mesh networks to detect and localize rain, see [7], [8]. However, most of the existing algorithms either work only for certain topologies or require two channels with different frequencies to detect rain, which limit their generality and applicability. [9] suggests a rain detection algorithm based on the cross-correlation of the samples of the received signals at both transmitter and receiver. However, sampling over 15 minutes time window is required to reliably determine the rain presence. Considering the highcapacity backhaul links, such a long delay can introduce a huge loss of traffic.

Adaptive power allocation [10] and link diversity [11] are proposed to compensate for the rain effect on a link between Macro Base Station (MBS) and satellite. However, these approaches are carried out locally (e.g., in a certain link), since they are designed to deal with the satellite communication. Consequently, they lead to suboptimal solution when applied in a mesh topology. Rerouting, as a new network- 
wide approach to relieve the rain effect, is considered in [5]. The authors propose two novel algorithms using physical-layer information expressed by BER to detect the rain presence and then to optimize routing at the network layer. Still, many aspects have not been well studied yet, such as the accuracy of the rain detector (e.g., distinguishing multipath and rain fading), performance degradation due to imperfect rain detection, and the overhead imposed to the network controller, which has an important impact on the network performance.

In this paper, we propose a novel robust rain detection algorithm to distinguish between short-term and long-term fading. In particular, we focus on the rain fading, albeit the proposed detection algorithm is quite general and can be readily extended to detect the long-term changes caused by the other reasons (e.g., obstacle in millimeter wave communications). We identify two types of detection errors, which may cause different network performance degradation, and we minimize both of them simultaneously by selecting proper parameters such as number of samples, number of voting links, and directionality. To investigate the impact of the detection algorithm, we also formulate a maximum flow problem that is handled by the central network controller. The results confirm that the proposed rain detection scheme can significantly reduce the huge traffic loss due to the rain fading with a very low overhead at the CC.

The remaining of the paper is organized as follows. In Section II, both the channel and network models are presented. Section III explains the rain detection algorithm that triggers the traffic rerouting function of the CC. The impact of the detection error on the network performance such as throughput and overhead imposed to the control plane is investigated in Section IV. Numerical results are presented in Section V. Finally, the conclusion is drawn in Section VI.

\section{Channel And Network Model}

We consider a partial mesh topology for the backhaul network in which some of MBSs are connected to each other, either directly or through some intermediate MBSs. Each MBS has a large number of antenna elements, making it feasible to create beams with very narrow beamwidth, known as pencilbeams [12]. We assume that the connections between every pair of MBSs are realized with the pencil-beams point-to-point line of sight (LoS) communications having negligible mutual interference among MBSs [12]. There is one edge node that aggregates the traffic and relays it to the core network (see an example shown in Fig. 1).

\section{A. Channel Model}

Depending on the weather condition, clear or rainy sky, the channels between MBSs would be affected by the multipath or rain fading. With pencil-beam operation, received power affected by multipath fading can be modeled using a Nakagami distribution. Let $X_{i}$ be the received power in $\mathrm{dB}$, considering the multipath fading experienced by link $i . X_{i}$ has the log-

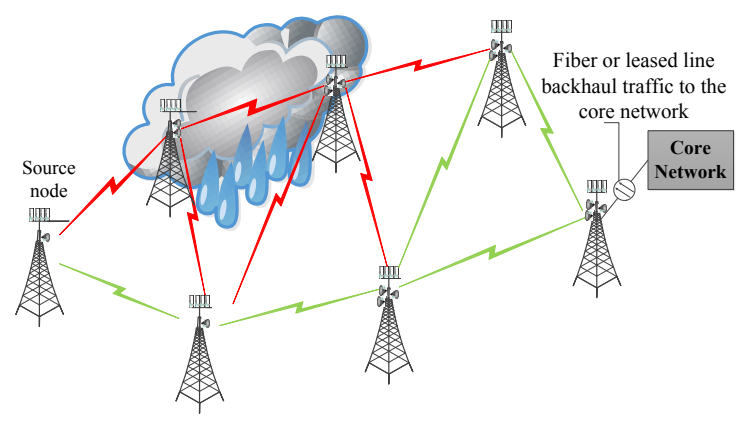

Figure 1. Topology of the network which is partially affected by rain (Red links: Affected links by rain; Green links: Non affected links)

Nakagami distribution [13]:

$$
f\left(X_{i}\right)=\frac{2 m^{m}}{M \Gamma(m) \bar{p}_{r, i}^{m}} \exp \left(\frac{2 m X_{i}}{M}-\frac{m}{\bar{p}_{r, i}} \exp \frac{2 X_{i}}{M}\right),
$$

where $M=20 \log (e), m$ and $\bar{p}_{r, i}$ represent the shape parameter (directionality) and the average received power for link $i$, respectively.

The rain fading at link $i$ in $\mathrm{dB}$, denoted by $A_{i}$, can be modeled with a Lognormal distribution [11], [14]:

$$
A_{i} \sim \ln N\left(n_{i}, \sigma_{i}\right)
$$

where $n_{i}$ and $\sigma_{i}$ are mean and standard deviation of $\ln \left(A_{i}\right)$. Considering that the rain fall is coherent along the entire radio path the mean is achieved by $n_{i}=\ln \left(\gamma R^{\eta} d_{i}\right)$, where $\gamma$ and $\eta$ are mainly functions of the frequency and polarization and $d_{i}$ is the length of $i$ th link [7]. The rain fading shows space and time correlation. Temporal correlation of the rain fading is modeled by autocorrelation function between time samples as [15]

$$
\gamma(\tau)=\frac{\exp \left(\sigma_{i} \exp (-\beta|\tau|)\right)-1}{\exp \left(\sigma_{i}\right)-1}
$$

where $\sigma_{i}$ is the standard deviation of $\ln \left(A_{i}\right)$ and $\beta$ describes the temporal variation of the rain attenuation on a link. To model the spatial correlation, [10] defines the concept of Correlated Area (CA) as the geographical area within which channel gains are highly correlated. This paper provides a way to find the CAs based on received signal to noise ratio (SNR). Besides, the spatial correlation factor from [16] can also be used to derive these CAs such that the set of the links with a high correlation factor establish one CA. We assume that the whole area can be divided into $|\mathrm{CA}|$ non-overlapping CAs. Let $\mathcal{V}$ and $\mathcal{E}$ represent the set of MBSs and the set of all communication links between MBSs, respectively. $\mathcal{C}_{i}$ denotes the set of MBSs in $\mathrm{CA}_{i}$, and we consider $\mathcal{C}_{i} \cap \mathcal{C}_{j}=\Phi$ and $\mathcal{C}_{i} \cup \mathcal{C}_{j}=\mathcal{V}$. We assume that the sets of $\mathcal{C}_{i}, i=1,2, \ldots,|\mathrm{CA}|$ are given a priori. Furthermore, $\mathcal{C}_{\mathcal{R}^{+}}$denotes the set of CAs that are affected by rain and $\mathcal{C}_{\mathcal{R}^{-}}$denotes the rest of CAs in which we have a clear sky. Based on existing experimental models of rain, we can assume that CAs belonging to $\mathcal{C}_{\mathcal{R}^{+}}$are randomly placed in the coverage area, matching the first-order statistical properties of rain [10].

\section{B. Network Model}

Let graph $G(\mathcal{V}, \mathcal{E})$ denote the topology of the network, where each vertex $i \in \mathcal{V}$ represents an MBS and each edge 
$(u, v) \in \mathcal{E}$ shows a communication link between MBSs $u$ and $v$. We consider a capacity $c_{u v}$ of every edge $(u, v) \in \mathcal{E}$, and a commodity $K$ originated from MBS $s$ with demand size $d$ destined to edge MBS $t$. The $f_{u v}$ denotes the flow along edge $(u, v)$ which should meet the following constraints [17]

$$
\begin{aligned}
& \sum_{\omega \in \mathcal{V}, u \neq s} \leqslant c_{u v}, \\
& \sum_{u \omega}-\sum_{\omega \in \mathcal{V}, k \neq t} f_{\omega k}=0, \forall u, k \\
& f_{s \omega}=\sum_{\omega \in \mathcal{V}} f_{\omega t}=d,
\end{aligned}
$$

where Constraint (4a) guarantees that the flows in each link $(u, v)$ cannot be bigger than the capacity of the link, Constraint (4b) shows that intermediate vertices are only relays and Constraint $(4 \mathrm{c})$ guarantees all the demands are served. In this paper, the objective is to maximize the throughput of the whole system. Hence, the objective function can be defined as

$$
\operatorname{maximize} \sum_{\omega \in \mathcal{V}} f(s, \omega) .
$$

The inputs to this optimization problem are traffic demand matrix, capacities of the individual links, and network topology $G$, whereas the outputs are the maximum flow that can be offered to MBS $s$ as well as corresponding route to the edge MBS. This analysis can be readily extended to the multicommodity maximum flow problem, which is the subject of our future studies. The CC of the backhaul network is responsible for solving this optimization problem periodically as some inputs need to be dynamically updated, e.g., due to the temporal variations of link capacity (a result of channel fading). In principle, this method can be also applied to respond to the short-term fluctuations of the channel gain. However, gathering all required inputs as frequent as shortterm channel fading occurs may impose huge overhead on the control plane, especially for the pencil-beam operation with high beam-training overhead [18]. It means that we should keep re-executions of this optimization problem as low as possible. Therefore, we believe that this global optimization is more proper to handle long-term channel attenuation, namely the rain fading, which is described in detail in the next section.

\section{RAin Detection Algorithm}

In this section, we propose a novel and relatively simple rain detection algorithm that leverages the time correlation between samples.

To detect the presence of rain in any $\mathrm{CA}$, we can define a binary variable $H_{j}$ for every $\mathrm{CA}_{j}$, taking 0 (or 1 ) as the absence (or presence) of rain on that $\mathrm{CA}$ :

$$
H_{j}= \begin{cases}0, & \text { if } \mathrm{CA}_{j} \in \mathcal{C}_{\mathcal{R}^{-}}, \\ 1, & \text { if } \mathrm{CA}_{j} \in \mathcal{C}_{\mathcal{R}^{+}} .\end{cases}
$$

Let $p_{r, i, j}(k)$ be the received power of link $i$ in $\mathrm{CA}_{j}$ at time slot $k$. Considering (6), we obtain

$$
p_{r, i, j}(k)[\mathrm{dB}]= \begin{cases}X_{i j}, & \text { if } \mathrm{CA}_{j} \in \mathcal{C}_{\mathcal{R}^{-}}, \\ X_{i j}-A_{i j}, & \text { if } \mathrm{CA}_{j} \in \mathcal{C}_{\mathcal{R}^{+}}\end{cases}
$$

where $X_{i j}$ and $A_{i j}$ are the received power and rain attenuation on link $i$ located in $\mathrm{CA}_{j}$, respectively. To solve this binary hypothesis testing problem, we first define the coherence time of the channel fading as the time interval within which the changed channel gain caused by this fading remains coherent. The coherence time of the multipath fading is much smaller than that of rain fading [19]. We assume that the channel attenuation is measured periodically with interval $\Delta$. Considering interval $\Delta$ bigger than the coherence time of the multipath fading process, the samples in $\left(H_{j}=0\right)$ hypothesis would be independent, whereas the samples from $\left(H_{j}=1\right)$ hypothesis would show significant correlation.

We define bad state as a situation in which the local action is not sufficient and rerouting is required, e.g., in the case of rain occurrence. Paper [16] suggests that bad state should be declared once $N$ consecutive samples taken from the channel attenuation become below a certain threshold (hereafter called as bad samples). However, due to the contribution of random multipath fading in every sample of the channel attenuation, this solution may not be accurate. Because, there is a chance of having the gain of one random channel higher than the threshold because of the multipath fading. To alleviate this problem, we suggest to declare rain (bad state) upon observing $K=\rho N$, where $\rho$ is an arbitrary value between $[0,1]$, bad samples in a window of $N \geq K$ consecutive samples of the channel gain. Inspired by $K$-out-of- $N$ detection rule that is widely adopted in spectrum sensing in cognitive radio networks [20], we propose the following rain detection procedure:

1) Each MBS defines a sample set of size $N$ keeping the last non overlapping $N$ samples of the channel condition.

2) Each MBS checks the received power with a predefined threshold $T$ and adds a bad sample in the sample set if the received power is below the threshold.

3) Upon gathering each $N$ new samples, the MBS checks the number of bad samples in the sample set. If $K$ samples in the set are bad samples, the MBS announces the existence of rain to the central controller and asks for rerouting.

To evaluate the performance of this detection procedure, we use the notion of false alarm and misdetection probabilities. False alarm is the one of being in the no-rain state but declaring the rain state, while misdetection is the one of being in the rain state but declaring no-rain state. With each misdetection, rerouting is not triggered, resulting in network performance degradation. With each false alarm, the central controller may unnecessarily execute a costly rerouting.

The false alarm event occurs once $K$ bad samples appear in $N$ consecutive samples in $\mathrm{CA}_{j} \in \mathcal{C}_{\mathcal{R}^{-}}$. Consider a window of $N$ consecutive samples. Every sample in this window is a Bernoulli random variable becoming bad sample with probability $\phi_{\mathrm{fa}, i, j}=\operatorname{Pr}\left[p_{r, i, j} \leqslant T \mid H_{j}=0\right]$. Assuming that the rain rate is constant during the window of $N$ samples $\phi_{\mathrm{fa}, i, j}$ becomes constant for all $N$ samples. Therefore, as the samples are independent and identically distributed, the probability of finding at least $K$ bad samples inside this window can be modeled by a summation of Binomial random 
variables with success probability $\phi_{\mathrm{fa}, i, j}$. Thus, we obtain

$$
\begin{aligned}
\phi_{\mathrm{fa}, i, j} & =\operatorname{Pr}\left[p_{r, i, j} \leqslant T \mid H_{j}=0\right] \\
& =\int_{0}^{T}\left(\frac{m}{\bar{p}_{r, i, j}}\right)^{m} \frac{x^{m-1}}{\Gamma(m)} \exp \left(\frac{-m x}{\bar{p}_{r, i, j}}\right) d x \\
& =1-\frac{\Gamma\left(m, \frac{m T}{\bar{p}_{r, i, j}}\right)}{\Gamma(m)},
\end{aligned}
$$

where $\Gamma(\cdot, \cdot)$ is the incomplete gamma function, and $\bar{p}_{r, i, j}$ is the average received power on link $i$ located in $\mathrm{CA}_{j}$. The false alarm probability of MBS $i$ at $\mathrm{CA}_{j} \in \mathcal{C}_{\mathcal{R}^{-}}$is

$$
p_{\mathrm{fa}, i, j}=\sum_{l=K}^{N}\left(\begin{array}{c}
N \\
l
\end{array}\right) \phi_{\mathrm{fa}, i, j}^{l}\left(1-\phi_{\mathrm{fa}, i, j}\right)^{N-l} .
$$

To increase the detection accuracy, we consider the spatial cooperation among MBSs inside a given CA. Taking into account a simple AND rule [20], the CC assumes that we have rain in $\mathrm{CA}_{j}$ provided that all MBSs in that CA report the existence of rain. Therefore, given a window of $N$ samples, the ultimate false alarm probability at the central controller for $\mathrm{CA}_{j}$, denoted by $P_{\mathrm{fa}, j}$, is given by

$$
P_{\mathrm{fa}, j}=\prod_{i \in \mathcal{C}_{j}} p_{\mathrm{fa}, i, j} .
$$

Using a similar approach, we can derive the misdetection probability. The success probability of the Bernoulli random variable, which models the misdetection, is equal to the probability that the received power is above the threshold when there is rain, namely

$$
\begin{aligned}
\phi_{\mathrm{md}, i, j} & =\operatorname{Pr}\left\{p_{r, i, j} \geqslant T \mid H_{j}=1\right\} \\
& =\int_{0}^{\infty} \frac{\Gamma\left(m, \frac{m \exp \left(\frac{2}{M}(T+y)\right)}{\bar{p}_{r, i, j}}\right)}{\Gamma(m)} f_{A_{i j}}(y) \mathrm{d} y,
\end{aligned}
$$

where $f_{A_{i j}}(y)$ is the probability density function of the rain attenuation. This integral does not have any closed-form, and we compute it numerically. As the rain rate is constant during the window of $N$ samples, $\phi_{\mathrm{md}, i, j}$ also becomes constant for all $N$ samples of the window. We can define the misdetection probability for link $i$ located in $\mathrm{CA}_{j}$ when the number of bad samples becomes less than $K$. The misdetection probability is the summation of the Binomial random variables as before, but the difference is in terms of the correlation between the samples. The summation of the correlated Binomial variables can be found in [21]. In this paper, the detailed mathematical computation is not presented, and we evaluate the performance of the system by simulation. Let us define $p_{\mathrm{md}, i, j}$ as a local misdetection probability, calculated according to [21]. Consider similar AND rule to combine decisions of different MBSs in $\mathrm{CA}_{j} \in \mathcal{C}_{\mathcal{R}^{+}}$. A misdetection occurs in the central controller on $\mathrm{CA}_{j}$ when at least one of the links located inside $\mathrm{CA}_{j}$ declares a clear sky. Hence, the misdetection probability for $\mathrm{CA}_{j} \in \mathcal{C}_{\mathcal{R}^{+}}$is

$$
P_{\mathrm{md}, j}=1-\prod_{i \in \mathcal{C}_{j}}\left(1-p_{\mathrm{md}, i, j}\right) .
$$

From (8)-(12), the performance of the rain detection is a function of the size of sampling window $N$, the number of bad samples for the rain declaration $K$, the threshold on the received power $T$, and the shape parameter of the Nakagami distribution $m$ (referred to as the directionality). Although we explain only the AND and OR rules for making the global decision in this section, we can use the general " $l$ out-of- $n$ " voting rule. That is, rain is declared if $l$ of the $n$ links detect rain. The performance of the proposed rain detection mechanism with respect to different voting rules is quantitatively investigated in Section V.

Increasing $N$ reduces both misdetection and false alarm probabilities, as the difference between correlated samples of channel attenuation (rain condition) and independent ones (clear sky) becomes more distinguishable. To capture the impact of the directionality on the rain detector performance, we have the following remarks:

Remark 3.1: Consider Equations (8) and (9). The false alarm probability goes to zero as $m$ grows large if $T \leq \bar{p}_{r, i, j}$.

Remark 3.2: Consider Equation (11). The misdetection probability goes to zero as $m$ grows large if $T \geq 10^{\frac{M}{20} \ln \bar{p}_{r, i, j}-\frac{n_{i}}{10}}$, where $\bar{p}_{r, i, j}$ and $n_{i}$ are the average received power and the average rain attenuation for link $i$ located in $\mathrm{CA}_{j}$.

These remarks provide bounds for proper values of $T$. For $T \in\left[10^{\frac{M}{20} \ln \bar{p}_{r, i, j}-\frac{n_{i}}{10}}, \bar{p}_{r, i, j}\right]$, we can improve the detection accuracy by using narrower beams. However, for the threshold values outside that bound increasing $m$ will improve either $P_{\mathrm{fa}}$ or $P_{\mathrm{md}}$ while deteriorating another one.

\section{IMPACt OF THE Detection ERRor on NetWork PERFORMANCE}

In this section, we study the effect of the rain detection error on the network throughput. Besides, the overhead that is caused by false alarm is also investigated.

To have better insight in the effect of false alarm and misdetection on the network performance, we propose the following analytical framework. Recall notation $\mathcal{C}_{\mathcal{R}^{-}}$and $\mathcal{C}_{\mathcal{R}^{+}}$ for the set of CAs with clear sky and rain, respectively. The rain occurrence notification caused by the rain detection at the MBSs located in $\mathcal{C}_{\mathcal{R}^{+}}$are received by the CC along with the false alarm notifications which are initiated by mistake by other MBSs in $\mathcal{C}_{\mathcal{R}^{-}}$. The rate of the request from $\mathcal{C}_{\mathcal{R}^{+}}$and $\mathcal{C}_{\mathcal{R}^{-}}$are equal to the detection and false alarm probabilities, respectively. Consequently, the total rerouting request rate $\lambda$ would be given by:

$$
\lambda=\sum_{j \in \mathcal{C}_{\mathcal{R}^{+}}}\left(1-P_{\mathrm{md}, j}\right)+\sum_{j \in \mathcal{C}_{\mathcal{R}^{-}}} P_{\mathrm{fa}, j},
$$

where $P_{\mathrm{fa}, j}$ and $P_{\mathrm{md}, j}$ are given in (10) and (12). The total overhead due to the false alarm probability is given by:

$$
\lambda_{e}=\sum_{j \in \mathcal{C}_{\mathcal{R}^{-}}} P_{\mathrm{fa}, j},
$$

where $\lambda_{e}$ shows the rerouting request rate caused by the false alarm.

The throughput gain TG achieved by using the proposed 


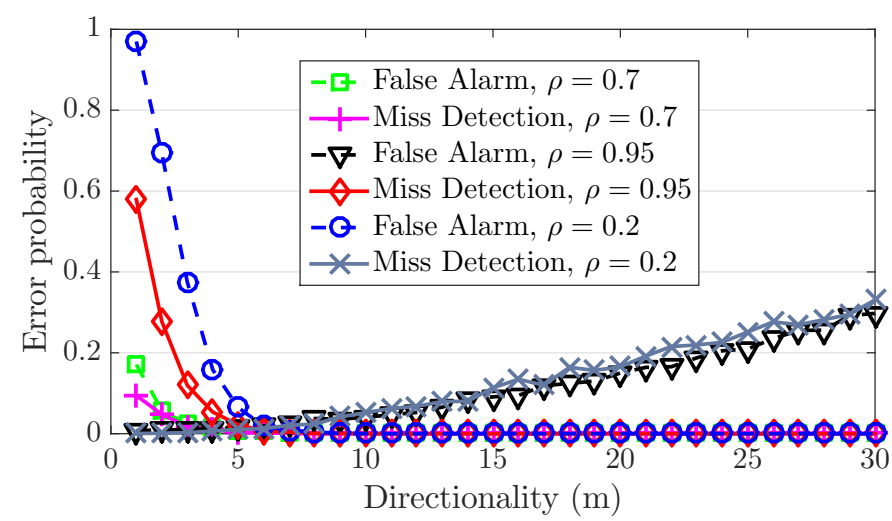

Figure 2. The impact of directionality $m$ on the misdetection and false alarm probabilities.

rain detection method can be computed as follow:

$\mathrm{TG}=\frac{1}{T-N \Delta t} \int_{N \Delta t}^{T}\left(1-\sum_{j \in \mathcal{C}_{\mathcal{R}^{+}}} P_{\mathrm{md}, j}\right) R(t)-R_{\mathrm{AMC}}(t) \mathrm{d} t$,

where $R$ represents the maximum achievable throughput when both the link adaption and rerouting are used and $R_{\mathrm{AMC}}$ is throughput when only the link adaption is implemented (e.g., $\mathrm{AMC}$ ). The throughput gain may decrease when the $\mathrm{CC}$ does not reroute in the presence of rain. Hence accurate detection would improve TG. Increasing $N$ improves the detection accuracy, but it takes longer time to identify rain, resulting in a huge loss of traffic during the detection. Due to this tradeoff, it is important to find a proper value of $N$ to achieve the acceptable detection accuracy while maintaining the detection process short in order to avoid high traffic loss.

\section{Performance Evaluation}

In this section, we numerically evaluate the performance of the proposed algorithm. The carrier frequency and transmitted power from each MBS are assumed $30 \mathrm{GHz}$ and $21 \mathrm{dBm}$, respectively, according to the commonly used value in the current microwave backhaul networks such as MINILINK [22]. Rain samples in time are generated according to the time autocorrelation function defined in Equation (3) with $\beta=1 / 9$ min. The mean and variance of the rain attenuation refer to [14], where $\gamma=1.07, \eta=1.04$ and $\sigma_{i}=1.4$. To measure the performance of our rain detector, we define weighted sum error as $P_{e}=P\left(H_{j}=0\right) P_{\mathrm{fa}}+P\left(H_{j}=1\right) P_{\mathrm{md}}$, where $P\left(H_{j}=0\right)$ and $P\left(H_{j}=1\right)$ are the probabilities of having clear sky and rainy weather, respectively. In this paper, we assume that the probability of the rain occurrence is equal to the one of the clear sky, so $P\left(H_{j}=0\right)=P\left(H_{j}=1\right)=0.5$. We consider mesh topology with 6 affected links as shown in Fig. 1 and rain rate for the area is $20 \mathrm{~mm} / \mathrm{h}$. Without loss of generality, in the local decision, we only consider one link, whereas all 6 affected links participate for the global decision handled by the central controller.

Fig. 2 illustrates Remarks 3.1 and 3.2. To achieve each point in Fig. 2, the optimal threshold that minimizes the total

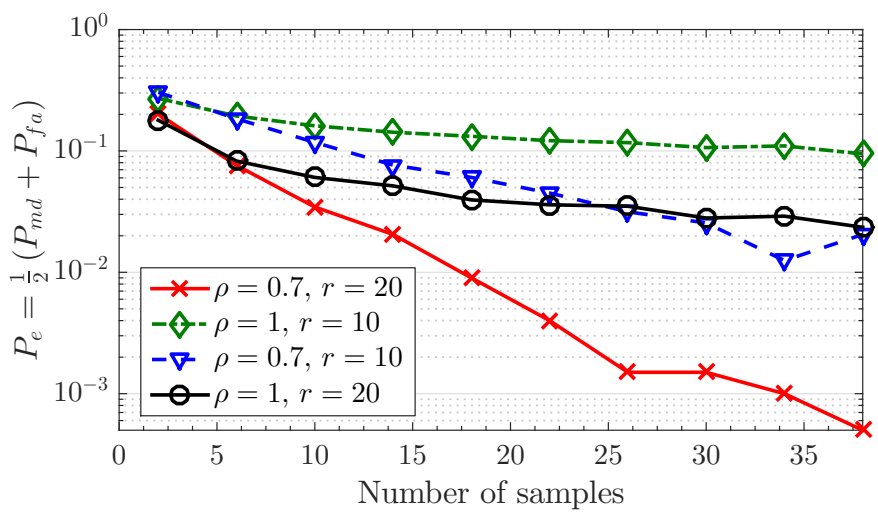

Figure 3. Total error probability versus $N$ for different rain rate shown as $r$.

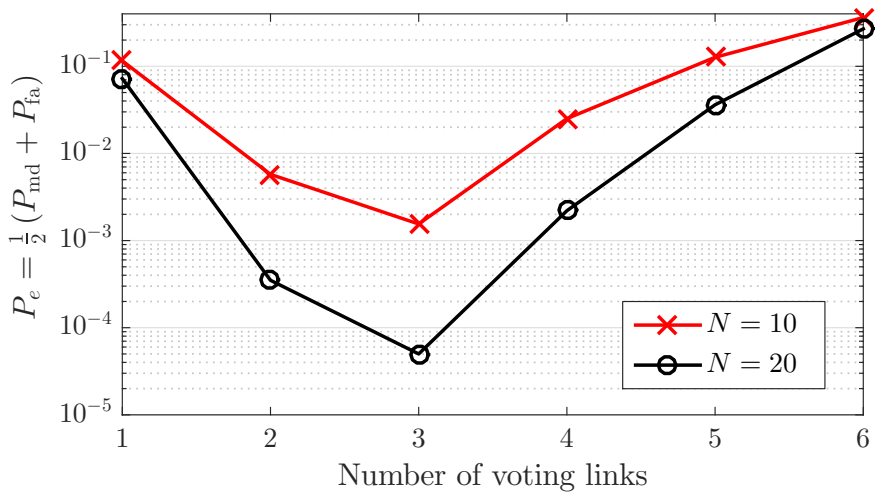

Figure 4. Impact of different voting rule on the total error probability for different $N$ when $m=5$.

error probability is calculated. From Fig. 2, when $\rho=0.7$ for which the optimal threshold is inside the bounds given by Remarks 3.1 and 3.2, increasing directionality decreases both the misdetection and false alarm probabilities. However, when $\rho=0.95$, the misdetection probability increases with $m$, as the optimal threshold when $\rho=0.95$ is higher than the boundary defined in Remark 3.2. Similar pattern can be observed also when $\rho=0.2$ for which the optimal threshold does not satisfy the inequality calculated in Remarks 3.1 and 3.2.

Fig. 3 illustrates the effect of the number of samples on $P_{e}$ for different $\rho$ and rain rate. We minimize $P_{e}$ by finding a proper value of $T$, while fixing all other parameters. Higher $N$ improves the detection performance. Note that, while not depicted in the figure, both misdetection and false alarm probabilities are decreased with $N$. Using a proper value for $\rho$ (e.g., $\rho=0.7$ in this example) significantly decreases the sum error. On the other hand, $\rho=1$ introduces a large detection error due to the randomness in multipath fading. As explained in Section III, the probability that all $N$ samples experience high channel attenuation is very low due to the independence of the multipath fading over different samples. Moreover, the detection performance would be slightly degraded if the rain rate is low.

The behavior of total error (i.e., $P_{e}=\frac{1}{2} P_{\mathrm{md}}+\frac{1}{2} P_{\mathrm{fa}}$ ) in global decision versus different voting rules is shown in Fig. 4. 


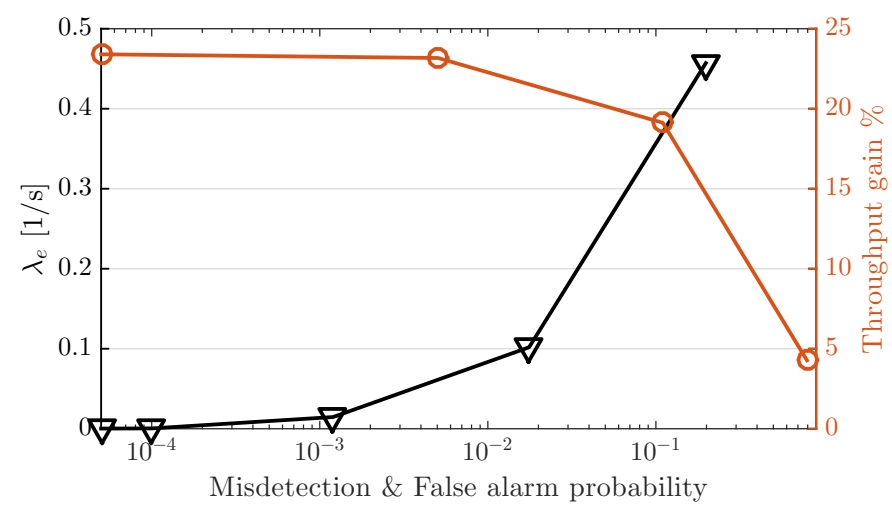

Figure 5. The effect of misdetection probability on the throughput gain, and the effect of false alarm probability on the overhead exposed to the $\mathrm{CC}$ when $N=10, m=5$.

It can be inferred that $P_{e}$ would be minimized when 3 out of 6 links detect rain. Moreover, spatial cooperation leads to a significant reduction in the detection error, compared to the local decision case. For example, cooperative detection reduces the error probability by $95 \%$ when $N=10$. This figure also emphasizes that the detection errors in the global decision would decrease for higher values of $N$.

In addition to the performance evaluation of the rain detector, we study the impact of imperfect rain detection on the throughput. We consider the same partial mesh topology as in Fig. 1 with one source and one destination to forward the traffic to the core network. We assume that rain affects a part of the network and lasts for the whole simulation time. In addition, we assume that the source node generates the traffic at $90 \mathrm{Mbps}$.

Throughput gain and $\lambda_{e}$ as a function of the misdetection and false alarm probabilities are depicted in Fig. 5. It can be inferred that high misdetection probability causes nonnegligible throughput reduction, since $\mathrm{CC}$ does not reroute the traffic once it is required. This figure also shows the overhead versus the false alarm probability. It is clear that increasing the false alarm probability imposes extra overheads on CC. These figures clarify the impact of imperfect rain detection on the network performance. Almost $16 \%$ throughput gain can be achieved by choosing an appropriate number of voting rule since it can reduce the detection error from 0.36 to 0.0016 , as shown in Fig. 4. Similarly, choosing a proper number of voting links, we can also substantially reduce the overhead.

\section{CONCLUSION}

This paper proposed a novel rain detection method and evaluated its accuracy by measuring false alarm and misdetection probabilities. A cooperative decision strategy was proposed, which can be carried out at the central controller, to take the optimal link adaptation action (either on the network level, e.g., rerouting, or only at the link level, e.g., adaptive modulation and coding) depending on the type of the channel fading. The results have verified that by choosing proper parameters, the unnecessary signaling and processing overhead of the central controller caused by the false alarms can be significantly reduced, while throughput reduction caused by the misdetection of rain presence can be eliminated.

\section{ACKNOWLEDGMENT}

The work described in this paper was carried out with the support of the Kista 5G Transport Lab (K5) project funded by VINNOVA, Ericsson AB, and Göran Gustafsson Grant.

\section{REFERENCES}

[1] J. Andrews et al., "What will 5G be?" IEEE Journal Selected Areas in Comm., vol. 6, no. 32, pp. 1065-1082, Jun. 2014.

[2] T. S. Rappaport et al., "Wideband millimeter-wave propagation measurements and channel models for future wireless communication system design," IEEE Trans. Commun., vol. 63, no. 9, pp. 3029-3056, Sept. 2015.

[3] S. Little, "Is microwave backhaul up to the 4G task?" IEEE Microwave Mag., vol. 10, no. 5, pp. 67-74, Aug. 2009.

[4] J. Salmelin and E. Metsälä, Mobile Backhaul. John Wiley \& Sons, 2012.

[5] A. Jabbar et al., "Performance comparison of weather disruption-tolerant cross-layer routing algorithms," in Proc. IEEE INFOCOM, 2009, pp. $1143-1151$.

[6] W. Xia et al., "A survey on software-defined networking," IEEE Commun. Surveys \& Tutorials, vol. 17, no. 1, pp. 27-51, First Quarter 2015.

[7] O. Goldshtein et al., "Rain rate estimation using measurements from commercial telecommunications links," IEEE Trans. Signal Proc., vol. 57, no. 4, pp. 1616-1625, April 2009.

[8] G. D. Abrajano and M. Okada, "Compressed sensing based detection of localized heavy rain using microwave network attenuation," in Proc. IEEE EUCAP, 2013, pp. 2383-2386.

[9] A. R. Rahimi et al., "Use of dual-frequency microwave links for measuring path-averaged rainfall," Journal of Geophysical Research: Atmospheres, vol. 108, no. D15, Aug. 2003.

[10] M. Castro and G. S. Granados, "Cross-layer packet scheduler design of a multibeam broadband satellite system with adaptive coding and modulation," IEEE Trans. Wireless Commun., vol. 6, no. 1, pp. 248258, Jan. 2007.

[11] M.Cheffena et al., "On the space-time variations of rain attenuation," IEEE Tran. Antennas and Propagation, vol. 57, no. 6, pp. 1771-1782, June 2009.

[12] S. Singh et al., "Interference analysis for highly directional $60-\mathrm{GHz}$ mesh networks: The case for rethinking medium access control," IEEE/ACM Trans. Netw., vol. 19, no. 5, pp. 1513-1527, Oct. 2011.

[13] H. Suzuki, "A statistical model for urban radio propogation," IEEE Trans. Commun., vol. 25, no. 7, pp. 673-680, Jul. 1977.

[14] S. Lin, "A method for calculating rain attenuation distributions on microwave paths," Bell System Technical Journal, vol. 54, no. 6, pp. 1051-1086, July-Aug. 1975.

[15] T. Maseng and P. M. Bakken, "A stochastic dynamic model of rain attenuation," IEEE Trans. Commun., vol. 29, no. 5, pp. 660-669, May 1981.

[16] B. C. Grémont and M. Filip, "Spatio-temporal rain attenuation model for application to fade mitigation techniques," IEEE Trans. Antennas Propagat., vol. 52, no. 5, pp. 1245-1256, May 2004.

[17] D. P. Bertsekas, Linear network optimization: algorithms and codes. MIT Press, 1991

[18] H. Shokri-Ghadikolaei et al., "Millimeter wave cellular networks: A MAC layer perspective," arXiv preprint arXiv:1503.00697, 2015.

[19] U.-C. Fiebig, "Modeling rain fading in satellite communications links," in Proc. IEEE VTC, vol. 3. IEEE, 1999, pp. 1422-1426.

[20] I. F. Akyildiz et al., "Cooperative spectrum sensing in cognitive radio networks: A survey," Physical Commun., vol. 4, no. 1, pp. 40-62, March 2011.

[21] A. Zaigraev and S. Kaniovski, "A note on the probability of at least k successes in n correlated binary trials," Operations Research Letters, vol. 41, no. 1, pp. 116-120, Jan. 2013.

[22] Ericsson AB, "MINI-LINK," [online]. Available: http: //www.ericsson.com/ourportfolio/products/microwave-networks?nav= productcategory004. 